\title{
Dermoscopic features of spider angioma in a healthy child
}

\section{Anissa Zaouak, Leila Bouhajja, Mariem Jrad, Amel Jebali, Houda Hammami, Samy Fenniche}

Dermatology Department, Habib Thameur Hospital, Tunis, Tunisia

Corresponding author: Dr. Anissa Zaouak, E-mail: anissa_zaouak@yahoo.fr

Sir,

We report a 7-year-old boy presented to our department for a newly appearing lesion of the cheek. He had no past medical history and there was not a history of trauma preceding the onset of the cutaneous lesion. Dermatologic examination revealed an erythematous small lesion with small vessels radiating from the center to the periphery located on the left cheek (Fig. 1). Dermoscopy revealed a vascular pattern with small telangiectasia and a stellate network (Fig. 2). The telangiectasias disappeared when pressure was applied with the dermoscope's glass (Fig. 3). The diagnosis of spider angioma was retained. An abdominal examination didn't reveal hepatomegaly or splenomegaly. Laboratory tests excluded the diagnosis of hepatitis, hepatic deficiency or cirrhosis. The patient was scheduled for a treatment with pulsed dye laser for his small vascular lesion.

Spider angiomas are lesions that appear as red spots, shiny, with numerous microvascular radiations which pale when pressure on a central spot is temporarily applied [1-2]. This condition was frequently associated with hepatic abnormalities [3]. Spider angiomas also called nevus araneus are lesions that appear as bright red small shiny lesions with numerous microvascular radiations which pale when pressure on a central spot is temporarily applied [1-3]. These lesions are made of small vessels radiating from center to peripheric zone. They are called spider angiomas because of their characteristic appearance resembling spider crotch. Nevus araneus has a small central artery that could pulsate. Several dilated vessels radiate from the central area. Compression of the central arteriole caused the

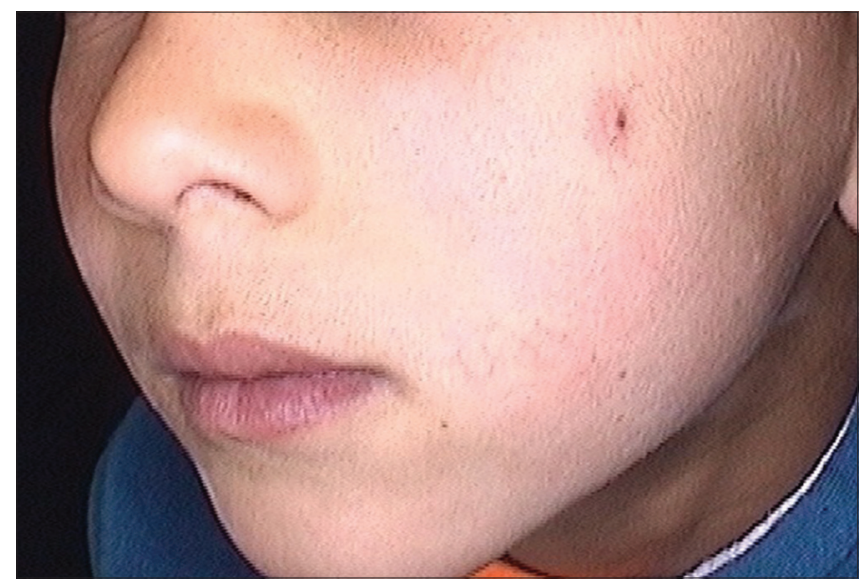

Figure 1: Small spider angioma on the left cheek in a child.

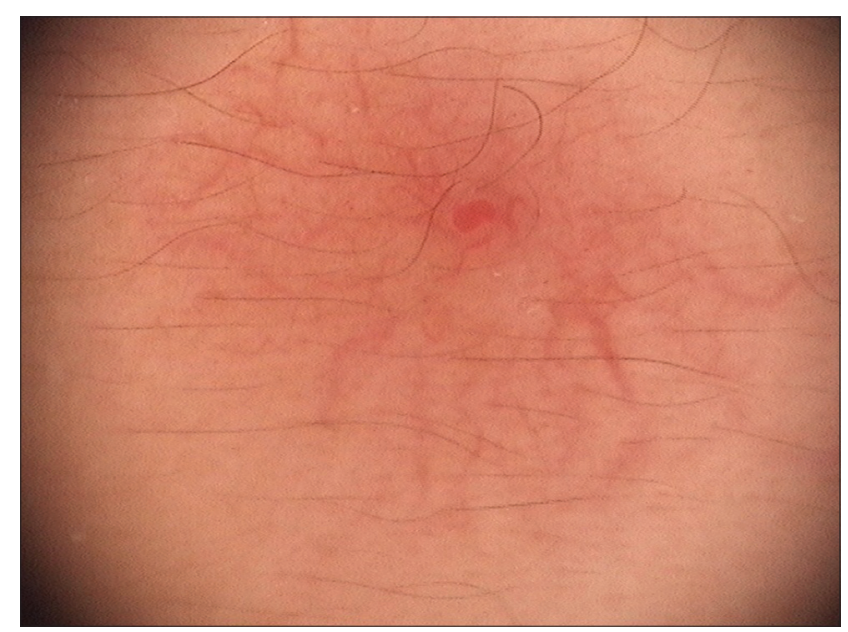

Figure 2: An arteriole in the central region that radiated out in numerous small vessels that resembled the legs of a spider.

entire lesion to blanch, and it quickly refilled once the compression was released. This pattern of blanching and refilling characterized spider angiomas [4]. The exact

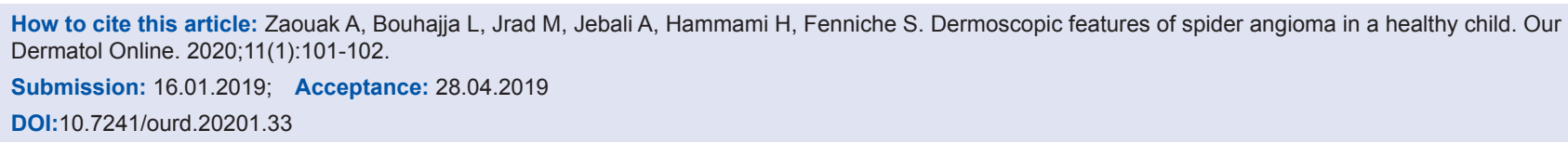




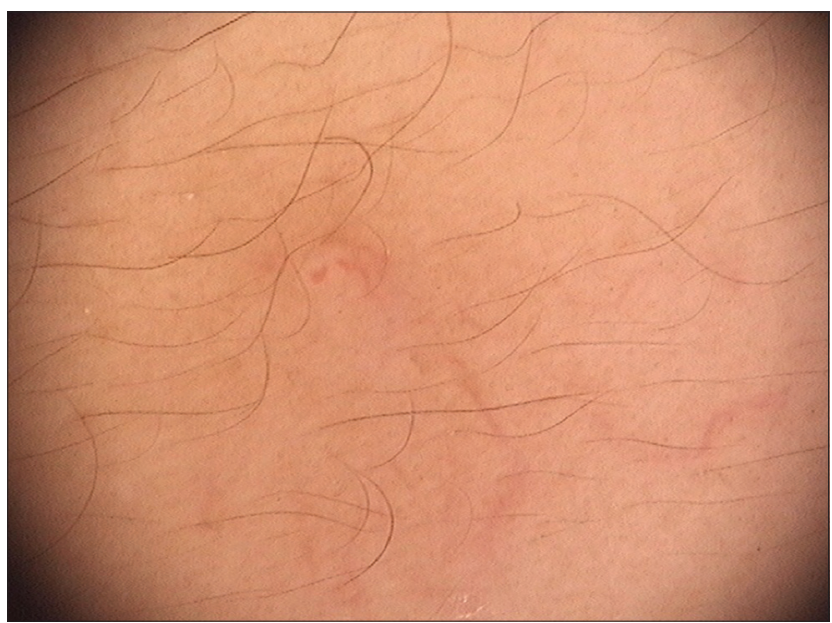

Figure 3: Compression of the central arteriole caused the entire lesion to blanch.

cause of spider angioma is not known. They may be present in physiological situations, such as in pregnancy, in children, in women under oral contraceptives, but are commonly associated with chronic liver diseases. Most frequent involvement sites of spider angiomas are the face, chest and arms. In our patient, the lesion was located on the face. Treatment of spider angiomas is not necessary. For cosmetic reasons, they could be treated using a fine electrolysis needle or with pulsed dye laser. However, sometimes spider angiomas may recur after treatment [3-5].
Spider angioma is a condition frequently associated with hepatic abnormalities. It can occur in children and has no pathologic signification. Dermoscopy could be helpful for the diagnosis of this facial vascular lesion.

\section{Consent}

The examination of the patient was conducted according to the Declaration of Helsinki principles.

\section{REFERENCES}

1. Finn SM, Rowland M, Lawlor F, Kinsella W, Chan L, Byrne O et al. The significance of cutaneous spider naevi in children. Arch Dis Child. 2006;91:604-5.

2. Samant H, Kothadia JP. Spider Angioma. StatPearls [Internet]. Treasure Island (FL): Stat Pearls Publishing; 2018 Jan-.2018 Oct 27.

3. Chuh A, Zawar V, Sciallis G. Does dermatoscopy facilitate the detection and diagnosis of vascular skin lesions? A case-control study. J R Coll Physicians Edinb. 2018;48:210-16.

4. Caseiro MM, da Costa SO. Images in clinical medicine. Spider angioma. N Engl J Med. 2012;366:e13.

5. Enta T. Dermacase. Nevus araneus (spider telangiectasia). Can Fam Physician. 1994;40:1105-12.

Copyright by Anissa Zaouak, et al. This is an open-access article distributed under the terms of the Creative Commons Attribution License, which permits unrestricted use, distribution, and reproduction in any medium, provided the original author and source are credited.

Source of Support: Nil, Conflict of Interest: None declared. 